\title{
Anafilaxia no primeiro ano de vida: como diagnosticar
}

\author{
Anaphylaxis in the first year of life: how to diagnose
}

\author{
Isabella Burla Manhães ${ }^{1}$, Carolina Sanchez Aranda ${ }^{2}$, Lucila de Camargo Oliveira², \\ Márcia Carvalho Mallozi',3, Gustavo Falbo Wandalsen², Dirceu Solé ${ }^{2}$
}

\section{RESUMO}

A anafilaxia é uma reação alérgica mais grave e potencialmente fatal. Apresenta-se quase sempre com manifestações cutâneas, acompanhadas por acometimento dos sistemas respiratório, gastrointestinal, nervoso e cardiovascular. Indivíduos de todas as faixas etárias podem manifestar anafilaxia, e seu diagnóstico no primeiro ano de vida é difícil por ser o lactente incapaz de expressar de modo claro as sensações vividas durante o episódio agudo. Nessa faixa etária os alimentos são os agentes desencadeantes mais envolvidos, embora medicamentos e veneno de himenópteros também o sejam. Em pacientes submetidos a várias cirurgias $e$ procedimentos médicos a alergia ao látex pode ocorrer. A adrenalina intramuscular é a primeira linha de tratamento da anafilaxia na fase inicial, mas continua sendo subutilizada. Além disso, medidas de suporte, tais como decúbito supino, reposição de fluidos, vias aéreas pérvias e oxigenação, devem ser instituídas. Após a alta, o paciente deve ser encaminhado à avaliação e seguimento por especialista visando à identificação do agente desencadeante, assim como educar responsáveis/cuidadores destes pacientes sobre a prevenção de novos episódios. É importante que esse paciente tenha consigo algum tipo de identificação que o aponte como tendo tido episódio de anafilaxia, sobretudo se tiver sido recorrente. A oferta de um plano escrito de como proceder diante de um novo episódio é fundamental.

Descritores: Anafilaxia, alimentos, medicamentos, veneno de insetos, epinefrina.

\begin{abstract}
Anaphylaxis is a serious and potentially fatal allergic reaction. Most frequently, it features cutaneous manifestations accompanied by involvement of the respiratory, gastrointestinal, nervous, and/or cardiovascular systems. Individuals of all age groups may present with anaphylaxis, and its diagnosis in the first year of life is difficult because the infant is unable to clearly express the sensations experienced during the acute episode. In this age group, foods are the most common triggering agents, together with medications and Hymenoptera venom. In patients undergoing multiple surgeries and medical procedures, latex allergy may occur. Intramuscular epinephrine is the first line of treatment for early anaphylaxis, but it remains underutilized. In addition, supportive measures such as supine decubitus, fluid replacement, patent airways, and oxygenation should be instituted. After discharge, the patient should be referred for evaluation and follow-up by a specialist, with the purpose of identifying the triggering agent as well as educating the caregivers of these patients about the prevention of new episodes. This patient should always carry some type of identification that indicates that he/she has had any episode of anaphylaxis, especially if it has been recurrent. Providing a written plan of how to proceed in the face of a new episode is essential.
\end{abstract}

Keywords: Anaphylaxis, food, medicine, insect venom, epinephrine.

\footnotetext{
1. Disciplina de Puericultura e Pediatria Social, Departamento de Pediatria, Escola Paulista de Medicina- Universidade Federal de São Paulo (EPM-UNIFESP) - São Paulo, SP, Brasil.

2. Disciplina de Alergia, Imunologia Clínica e Reumatologia, Departamento de Pediatria, EPM-UNIFESP - São Paulo, SP, Brasil.

3. Departamento Materno Infantil da Fundação Faculdade de Medicina do ABC - Santo André, SP, Brasil.
} 


\section{Introdução}

Nas últimas décadas várias definições foram propostas para a anafilaxia, umas simples ${ }^{1,2}$ e outras mais detalhadas que abordam mecanismos fisiopatológicos ${ }^{3}$ e/ou manifestações clínicas ${ }^{4-6}$. Assim, a anafilaxia é definida como uma reação de hipersensibilidade sistêmica grave com risco de vida, caracterizada por início rápido, e com potenciais problemas de vias aéreas, respiratórios ou circulatórios e geralmente, embora nem sempre, associada a alterações cutâneas e em mucosas ${ }^{5}$.

De modo geral, a anafilaxia em menores de um ano é de difícil diagnóstico, tendo-se em consideração as características do paciente (não verbal), e que várias situações clínicas podem mimetizar sinais e sintomas que podem estar presentes no lactente. Foram objetivos deste estudo revisar a literatura com relação ao comportamento da anafilaxia no primeiro ano de vida, destacando-se os agentes etiológicos, manifestações clínicas, tratamento e evolução e seguimento de longo prazo.

\section{Fonte de dados}

Revisão não sistemática da literatura, com busca de artigos desde 2005 no PubMed, Google Scholar e SciELO usando os termos "anaphylaxis" ou "severe allergic reaction" ou "severe food-induced allergic reaction" ou "epinephrine" e "infants" ou "toddlers", nos idiomas inglês, francês, espanhol e português.

\section{Critérios diagnósticos}

Embora os critérios clínicos diagnósticos para anafilaxia tenham sido estabelecidos inicialmente em $2006^{4}$, reforçados por outros grupos ${ }^{6,7}$ e venham sendo utilizados, o diagnóstico de anafilaxia ainda não é tarefa fácil, sobretudo em lactentes nos primeiros dois anos de vida. Recentemente, o Comitê de Anafilaxia da World Allergy Organization (WAO) atualizou e resumiu os critérios diagnósticos de anafilaxia ${ }^{8,9}$. A comparação entre os critérios iniciais e os da WAO são apresentados na Tabela 1.

\section{Epidemiologia}

A falta de um código internacional de doença (CID) específico para rotular as reações anafiláticas até o CID-10 em muito contribuiu para que os episódios de anafilaxia recebessem codificações variadas, na dependência das manifestações clínicas apresentadas, dificultando assim a obtenção de dados epidemiológicos mais consistentes em estudos retrospectivos e baseados em banco de dados ${ }^{10}$. Além disso, a homogeneidade dos estudos com relação às populações estudadas (população geral, pacientes hospitalizados, idas à unidade de urgência, entre outros), o emprego de definições precisas, de critérios consistentes para a identificação dos casos de anafilaxia (história clínica, testes laboratoriais), o subdiagnóstico, assim como a padronização do método estatístico para a obtenção das taxas de inicidência e de prevalência são pontos fundamentais que nos permitem obter dados passíveis de comparação e não são encontrados em grande parte dos estudos existentes ${ }^{11}$.

À semelhança do observado com as doenças alérgicas, a prevalência de anafilaxia tem aumentado nas últimas décadas. Estima-se que o mesmo ocorra entre os lactentes, embora nessa faixa etária as taxas reais ainda permaneçam desconhecidas. A frequência de anafilaxia entre lactentes atendidos em serviços de urgência tem oscilado entre 3\% e $22 \%{ }^{12-14}$. Os alimentos têm sido os principais agentes etiológicos responsabilizados pela anafilaxia nesses pacientes ${ }^{11-13}$. Apesar disso, há evidências de que estes episódios sejam menos graves entre os lactentes, quando comparados às crianças maiores ${ }^{15-18}$.

A Tabela 2 reúne alguns estudos sobre anafilaxia que avaliaram lactentes. Revisão sistemática avaliou a epidemiologia da anafilaxia em crianças (menores de 18 anos de idade) europeias e documentou, em 2013, ser a prevalência agrupada de anafilaxia $0,3 \%$ e que a incidência oscilou entre 1,5 e 32,0 casos por 100.000 indivíduos-ano ${ }^{19}$. Outra revisão sistemática reuniu 59 estudos em menores de 18 anos (registros de pacientes, atendimento em unidades de urgência e hospitalizações) de diferentes localidades do mundo e revelou ser a prevalência estimada de anafilaxia variável entre $0,04 \%$ e 1,8\% (4 estudos) e a por alimentos entre $0,3 \%$ e 1,2\% (10 estudos) $)^{11}$. As taxas de inicidência de anafilaxia (29 estudos) oscilaram entre 1 e 761 casos por 100.000 indivíduos/ano, e os alimentos são os principais responsáveis por esses índices (1 a 77 por 100.000 indivíduos/ano) ${ }^{11}$.

Na América Latina, os dados sobre a epidemiologia da anafilaxia são escassos, e muitas vezes limitados a grupos específicos de pacientes. O estudo OLASA (On-line Study of Anaphylaxis in Latinamerica) reuniu dados sobre pacientes atendi- 
Tabela 1

Critérios clínicos diagnósticos de anafilaxia

\section{Critérios clínicos}

Anafilaxia é altamente provável quando presente:

NIAID/FAAN ${ }^{4}$

pelo menos um dos três critérios
World Allergy Organization ${ }^{8,9}$

pelo menos um dos dois critérios
1 - Doença de início agudo (minutos a várias horas) com envolvimento simultâneo da pele, tecido mucoso ou ambos (por exemplo, urticária generalizada, prurido ou rubor, inchaço dos lábios-língua-úvula)

\section{E pelo menos um dos seguintes:}

a) comprometimento respiratório (por exemplo: dispneia, sibilância-broncoespasmo, estridor, redução do PFE, hipoxemia)

b) redução da PA ou sintomas de disfunção de órgão-alvo (por exemplo: hipotonia [colapso], síncope, incontinência)

\section{OU}

2 - Dois ou mais dos seguintes que ocorrem rapidamente após a exposição a um alérgeno provável para aquele paciente (minutos a várias horas)

a) envolvimento do tecido cutâneo-mucoso (por exemplo: urticária generalizada, prurido rubor, edema lábios-língua-úvula)

b) comprometimento respiratório (por exemplo: dispneia, sibilância-broncoespasmo, estridor, redução do PFE, hipoxemia)

c) redução da pressão sanguínea ou sintomas de disfunção de órgão-alvo (por exemplo: hipotonia [colapso], síncope, incontinência)

\section{OU}

3 - Redução da pressão sanguínea após exposição a alérgeno conhecido para um determinado paciente (minutos a várias horas)

a) lactentes e crianças: PA sistólica baixa (idade específico) ou maior que $30 \%$ de queda na PA sistólica ${ }^{a}$

b) adultos: PA sistólica abaixo de $90 \mathrm{mmHg}$ ou de queda de mais do que $30 \%$ do basal para aquela pessoa
1 - Doença de início agudo (minutos a várias horas) com envolvimento simultâneo da pele, tecido mucoso ou ambos (por exemplo, urticária generalizada, prurido ou rubor, inchaço dos lábios-língua-úvula)

\section{E pelo menos um dos seguintes:}

a) comprometimento respiratório (por exemplo: dispneia, sibilância-broncoespasmo, estridor, redução do PFE, hipoxemia)

b) redução da PA ou sintomas de disfunção de órgão-alvo (por exemplo: hipotonia [colapso], síncope, incontinência)

c) sintomas gastrointestinais intensos (por exemplo: cólica abdominal intensa, vômitos de repetição) especialmente após exposição a alérgenos não alimentares.

\section{OU}

2 - Hipotensãoa de início agudo ou broncoespasmob ou envolvimento laríngeo ${ }^{c}$ após exposição a alérgeno ${ }^{d}$ conhecido ou altamente provável para aquele paciente (minutos a várias horas), mesmo na ausência de acometimento cutâneo típico

NIAID = National Institutes of Allergy and Infectious Diseases, FAANS = Food Allergy and Anaphylaxis Network, PFE = pico de fluxo expiratório, $\mathrm{PA}=$ pressão arterial.

a Hipotensão definida como uma diminuição em PA sistólica maior que 30\% do valor basal dessa pessoa, OU i. Bebês e crianças menores de 10 anos: PA sistólica menor que $(70 \mathrm{mmHg}+[2 \mathrm{x}$ idade em anos]) ii. Adultos e crianças com mais de 10 anos: PA sistólica menor que $<90 \mathrm{mmHg}$.

b Excluindo sintomas respiratórios inferiores desencadeados por alérgenos inalantes comuns ou alérgenos alimentares considerados como causadores de reações "inalatórias" na ausência de ingestão.

c Os sintomas laríngeos incluem: estridor, alterações vocais, odinofagia.

d Alérgeno é uma substância (geralmente uma proteína) capaz de desencadear uma resposta imunológica que pode resultar em uma reação alérgica. A maioria dos alérgenos age através de mecanismo dependente de IgE, mas alguns desencadeantes não alérgenos podem agir independentemente da IgE (por exemplo, por meio da ativação direta dos mastócitos). 
dos por alergologistas de 14 países (América Latina e Portugal) por quadro de anafilaxia entre 2008 e 2010. Participaram da versão pediátrica 191 indivíduos menores de 18 anos, sendo que $16,3 \%$ eram menores de dois anos de idade. Nesse estudo os alimentos foram os principais desencadeantes, e $14,3 \%$ foram hospitalizados ${ }^{20}$.

Estudo realizado em hospital pediátrico na cidade de São Paulo avaliou a incidência de atendimentos em unidade de emergência de crianças e adoles- centes (até 18 anos), entre 2016 e 2018, por quadro de possível anafilaxia (CID-10) ${ }^{21}$. O número total de atendimentos em 2016, 2017 e 2018 foi 108.695, 105.523 e 102.133, respectivamente. As taxas de incidência de prováveis casos nos respectivos anos foram $0,011 \%, 0,013 \%$ e $0,016 \%$ respectivamente e a incidência para o período foi $0,013 \%$. Estudo polonês revelou ser a frequência de anafilaxia 0,3\% entre crianças ( 0 a 3 anos) hospitalizadas, e 1,9\% das com suspeita de alergia alimentar ${ }^{23}$.

\section{Tabela 2}

Exemplos de estudos que avaliaram anafilaxia em menores de dois anos ${ }^{15}$

\begin{tabular}{|c|c|c|c|c|c|}
\hline $\begin{array}{l}\text { Autores, } \\
\text { ano, país }\end{array}$ & $\begin{array}{l}\text { Tipo de } \\
\text { estudo }\end{array}$ & $\begin{array}{l}\text { População } \\
\text { total }\end{array}$ & $\begin{array}{l}\text { Distribuição } \\
\text { lactentes }\end{array}$ & $\begin{array}{l}\text { Sintomas } \\
\text { clínicos }\end{array}$ & $\begin{array}{l}\text { Agentes } \\
\text { desencadeantes }\end{array}$ \\
\hline $\begin{array}{l}\text { Ko et al., } \\
2020 \\
\text { EUA }^{16}\end{array}$ & $\begin{array}{l}\text { Retrospectivo } \\
\text { Casos atendidos em } \\
\text { unidade de urgência } \\
\text { (janeiro } 2016 \text { a } \\
\text { dezembro 2018) }\end{array}$ & $\begin{array}{l}\text { Crianças } \\
\text { menores } \\
\text { de } 5 \text { anos } \\
(n=1.518)\end{array}$ & $\begin{array}{l}<1 \text { ano } \\
(n=448)\end{array}$ & $\begin{array}{l}\text { MC }-99 \% * \\
\text { GI }-32 \% \\
\text { Resp - } 7 \% \\
\text { CV }-0,9 \% \\
\text { Hip }-0,2 \%\end{array}$ & $\begin{array}{l}\text { Ovo - } 34 \%^{*} \\
\text { Amendoim - } 22 \% \\
\text { LV - } 16 \%^{*} \\
\text { Nozes - } 8 \% \\
\text { Sementes - } 4 \% \\
\text { Peixes - 3\% }\end{array}$ \\
\hline $\begin{array}{l}\text { Pouessel et al., } \\
2020, \\
\text { França }^{17}\end{array}$ & $\begin{array}{l}\text { Retrospectivo } \\
\text { Allergy Vigilance } \\
\text { Network } \\
(2002-2018)\end{array}$ & $\begin{array}{l}\text { Crianças } \\
\text { menores } \\
\text { de } 6 \text { anos } \\
(n=375)\end{array}$ & $\begin{array}{l}\leq 1 \text { ano } \\
(n=59)\end{array}$ & $\begin{array}{l}\text { MC - } 79 \% * \\
\text { GI - } 49 \% \\
\text { Resp - } 48 \% \\
\text { (dispneia - } 31 \% \text {, } \\
\text { sibilância - } 11 \% \text {, } \\
\text { edema laringe }-11 \% \text {, } \\
\text { tosse }-10 \% \text { ) } \\
\text { Neuro }-26 \%{ }^{*} \\
\text { CV - } 21 \%{ }^{*}\end{array}$ & $\begin{array}{l}\text { LV - } 59 \%^{*} \\
\text { Ovo - } 20 \%^{*} \\
\text { Trigo - } 7 \% \\
\text { Amendoim - 3\% }\end{array}$ \\
\hline $\begin{array}{l}\text { Joen et al., } \\
2019, \\
\text { Koreia }^{24}\end{array}$ & $\begin{array}{l}\text { Retrospectivo } \\
\text { de hospitalizações } \\
\text { por anafilaxia } \\
\text { entre } 2009 \text { e } 2013\end{array}$ & $\begin{array}{l}\text { Lactentes } \\
<\text { de } 2 \text { anos } \\
\text { com quadro } \\
\text { de anafilaxia } \\
(n=363)\end{array}$ & $\begin{array}{l}<2 \text { anos } \\
(n=363)\end{array}$ & $\begin{array}{l}\text { MC - } 99 \% \\
\text { Resp - } 83 \% \\
\text { Gl - } 30 \% \\
\text { Neuro - } 12 \% \\
\text { CV - } 8 \%\end{array}$ & $\begin{array}{l}\text { LV - } 44 \% \\
\text { Ovo - } 22 \% \\
\text { Trigo - } 8 \% \\
\text { Nozes - } 8 \% \\
\text { Amendoim - 5\% } \\
\text { Castanhas - 3\% } \\
\text { Peixe - } 2 \% \\
\text { Soja - } 2 \% \\
\text { Drogas - 3\% } \\
\text { Exercício induzida } \\
\text { alimento - 0,9\% } \\
\text { Picada de inseto - } 0,6 \%\end{array}$ \\
\hline $\begin{array}{l}\text { Samady et al., } \\
2018 \text {, } \\
\text { EUA }^{25}\end{array}$ & $\begin{array}{l}\text { Retrospectivo } \\
\text { série de casos } \\
\text { atendidos em } \\
\text { unidade de urgência } \\
\text { junho } 2015 \text { a } 2017\end{array}$ & $\begin{array}{l}\text { Crianças } \\
(<18 \text { anos }) \\
\text { com anafilaxia } \\
\text { por alimento } \\
(n=357)\end{array}$ & $\begin{array}{l}<12 \text { meses } \\
(n=47)\end{array}$ & $\begin{array}{l}\text { MC - } 94 \%{ }^{*} \\
\text { GI - } 89 \% \\
\text { Resp - } 17 \% \\
\text { (dificuldade de } \\
\text { respirar-11\%; } \\
\text { tosse - } 9 \% \text {; } \\
\text { sibilância - } 2 \% \text { ) } \\
\text { Orofaringe - } 9 \% \\
\text { CV - } 2 \%\end{array}$ & $\begin{array}{l}\text { Ovo - } 38 \%^{*} \\
\text { LV - } 17 \%^{*} \\
\text { Amendoim - } 13 \% \\
\text { Frutos secos }-6 \%\end{array}$ \\
\hline
\end{tabular}

* Significantemente mais frequente entre os menores de 12 meses em comparação aos de outras faixas etárias.

$\mathrm{MC}=$ mucocutâneos, $\mathrm{Gl}$ = gastrointestinal, Resp = respiratório, CV = cardiovascular, Hip = hipotensão, ? = não calculável, Alt comp = alteração de comportamento, Ovo = ovo de galinha, LV = leite de vaca. 
Tabela 2 (continuação)

Exemplos de estudos que avaliaram anafilaxia em menores de dois anos ${ }^{15}$

\begin{tabular}{|c|c|c|c|c|c|}
\hline $\begin{array}{l}\text { Autores, } \\
\text { ano, país }\end{array}$ & $\begin{array}{l}\text { Tipo de } \\
\text { estudo }\end{array}$ & $\begin{array}{l}\text { População } \\
\text { total }\end{array}$ & $\begin{array}{l}\text { Distribuição } \\
\text { lactentes }\end{array}$ & $\begin{array}{l}\text { Sintomas } \\
\text { clínicos }\end{array}$ & $\begin{array}{l}\text { Agentes } \\
\text { desencadeantes }\end{array}$ \\
\hline $\begin{array}{l}\text { Topal et al., } \\
2013, \\
\text { Turquia }^{18}\end{array}$ & $\begin{array}{l}\text { Retrospectivo, } \\
\text { série de casos } \\
\text { (janeiro } 2007 \text { a } \\
\text { dezembro 2011) }\end{array}$ & $\begin{array}{l}\text { Crianças } \\
<17 \text { anos } \\
\text { com anafilaxia } \\
\text { documentada } \\
(n=104)\end{array}$ & $\begin{array}{l}\leq 12 \text { meses } \\
(n=23)\end{array}$ & $\begin{array}{l}\text { MC - 100\% } \\
\text { Resp - 78\% } \\
\text { (estridor - 48\%; } \\
\text { tosse - 30\%; } \\
\text { sibilância - 17\%; } \\
\text { rouquidão - 4\%) } \\
\text { GI - } 26 \% \\
\text { CV - ? }\end{array}$ & $\begin{array}{l}\text { Alimento - } 91 \%{ }^{*} \\
\text { LV - } 61 \% \\
\text { Ovo - } 22 \% \\
\text { Nozes - } 9 \%\end{array}$ \\
\hline $\begin{array}{l}\text { Fleisher et al., } \\
2012, \\
\text { EUA }^{26}\end{array}$ & $\begin{array}{l}\text { Multicêntrico, } \\
\text { prospectivo } \\
\text { observacional, } \\
36 \text { meses de } \\
\text { seguimento }\end{array}$ & $\begin{array}{l}\text { Lactentes } \\
\text { com reação } \\
\text { a alimentos } \\
(n=512)\end{array}$ & $\begin{array}{l}3 \text { a } 15 \text { meses } \\
(n=512)\end{array}$ & - & $\begin{array}{l}\text { LV - } 42 \% \\
\text { Ovo - } 21 \% \\
\text { Amendoim - } 8 \% \\
\text { Trigo - } 4 \% \\
\text { Peixe - 3\% } \\
\text { Frutos secos - 3\% }\end{array}$ \\
\hline $\begin{array}{l}\text { Rudders et al., } \\
2011, \\
E^{27}\end{array}$ & $\begin{array}{l}\text { Retrospectivo, } \\
\text { série de casos } \\
\text { atendidos em } \\
\text { unidade de urgência } \\
\text { (janeiro } 2001 \\
\text { a dezembro 2006) }\end{array}$ & $\begin{array}{l}\text { Crianças } \\
<18 \text { anos } \\
\text { atendidas } \\
\text { por anafilaxia } \\
\text { a alimento } \\
(n=522)\end{array}$ & $\begin{array}{l}<12 \text { meses } \\
(n=191)\end{array}$ & $\begin{array}{l}\text { MC - } 98 \%{ }^{*} \\
\text { Resp - } 59 \% \\
\text { GI - } 56 \% \\
\text { CV - ? }\end{array}$ & $\begin{array}{l}\text { LV - } 40 \%{ }^{*} \\
\text { Amendoim - 31\%* } \\
\text { Ovo - } 9 \% \\
\text { Frutos secos - } 9 \%\end{array}$ \\
\hline $\begin{array}{l}\text { Solé et al., } \\
2012 \text {, } \\
\text { América Latina } \\
\text { e Portugal }{ }^{20}\end{array}$ & $\begin{array}{l}\text { Retrospectivo } \\
\text { série de casos } \\
\text { com diagnóstico } \\
\text { de anafilaxia } \\
\text { atendidos por } \\
\text { alergologistas } \\
2006 \text { a } 2008\end{array}$ & $\begin{array}{l}\text { Crianças } \\
<18 \text { anos } \\
\text { com anafilaxia } \\
\text { documentada }\end{array}$ & $\begin{array}{l}<2 \text { anos } \\
(n=31)\end{array}$ & - & $\begin{array}{l}\text { Alimento }-53,3 \%^{*} \\
\text { Ovo }-25,8 \%^{*} \\
\text { LV }-22,6 \%{ }^{*} \\
\text { Frutas }-3,2 \%\end{array}$ \\
\hline $\begin{array}{l}\text { Alvarez-Perea } \\
\text { et al., } 2017 \text {, } \\
\text { Espanha }^{28}\end{array}$ & $\begin{array}{l}\text { Atendimentos } \\
\text { por anafilaxia } \\
\text { em unidade } \\
\text { de urgência } \\
\text { de hospital } \\
2012 \text { a } 2014\end{array}$ & $\begin{array}{l}\text { Crianças } \\
<18 \text { anos } \\
(\mathrm{n}=133) \\
\text { Incidência } \\
\text { cumulativa } \\
\text { de } 0,12 \%\end{array}$ & $\begin{array}{l}<1 \text { ano } \\
(n=20)\end{array}$ & - & $\begin{array}{l}\text { LV - } 67 \% \\
\text { Ovo - } 22 \% \\
\text { Frutas - } 6 \% \\
\text { Peixe - } 6 \%\end{array}$ \\
\hline $\begin{array}{l}\text { Kahveci et al., } \\
2020, \\
\text { Turquia } 29\end{array}$ & $\begin{array}{l}\text { Estudo } \\
\text { retrospectivo } \\
\text { de crianças } \\
\text { com anafilaxia } \\
\text { por alimento }\end{array}$ & $\begin{array}{l}\text { Crianças } \\
<6 \text { anos } \\
(n=314)\end{array}$ & $\begin{array}{l}<1 \text { ano } \\
(n=160)\end{array}$ & $\begin{array}{l}\text { Pele }-90,9 \% \\
\text { Resp - } 58,9 \% \\
\text { GI - } 56 \% \\
\text { Mucosa }-45,1 \% \\
\text { Neuro }-14,9 \%\end{array}$ & $\begin{array}{l}\text { LV - 51,4\%* } \\
\text { Nozes - 16,6\% } \\
\text { Ovo - 15,4\% } \\
\text { Trigo - 5,7\% } \\
\text { Lentilha - 5,1\% } \\
\text { Gergelim - } 4,6 \%\end{array}$ \\
\hline $\begin{array}{l}\text { Pistiner et al., } \\
2021 \\
\text { EUA }^{30}\end{array}$ & $\begin{array}{l}\text { Pesquisa online } \\
\text { nacional com } \\
\text { cuidadores de } \\
\text { crianças } \\
\text { que tiveram } \\
\text { anafilaxia grave }\end{array}$ & $\begin{array}{l}\text { Crianças } \\
\text { até } 36 \text { meses } \\
(n=374)\end{array}$ & $\begin{array}{l}<1 \text { ano } \\
(n=193)\end{array}$ & $\begin{array}{l}\text { Pele - } 94 \% \\
\text { Mucosa - 56,4\% } \\
\text { Gl - } 55 \% \\
\text { Resp - 38,9\% } \\
\text { Alt comp - 32,6\% } \\
\text { CV - } 15 \%\end{array}$ & $\begin{array}{l}\text { LV - } 30 \% \\
\text { Ovo - } 28 \% \\
\text { Amendoim - 17\% } \\
\text { Trigo - } 6 \% \\
\text { Nozes - } 4 \% \\
\text { Gergelim - 3\% } \\
\text { Soja - } 1 \% \\
\text { Fruta - } 1 \% \\
\text { Peixe - } 0,5 \%\end{array}$ \\
\hline
\end{tabular}

* Significantemente mais frequente entre os menores de 12 meses em comparação aos de outras faixas etárias.

$\mathrm{MC}=$ mucocutâneos, $\mathrm{Gl}=$ gastrointestinal, Resp= respiratório, $\mathrm{CV}=$ cardiovascular, Hip = hipotensão, ? = não calculável, Alt comp=alteração de comportamento, Ovo = ovo de galinha, LV = leite de vaca. 


\section{Fatalidades}

As fatalidades atribuídas à anafilaxia entre crianças são eventos raríssimos ${ }^{16-18,26}$. Segundo Tsuang e cols., até a atualidade conhece-se apenas o relato de um lactente de 5 meses de idade, alérgico ao leite de vaca e que desenvolveu anafilaxia fatal ao ingerir cereal que continha leite de vaca na sua composição ${ }^{15,31}$.

Metanálise recente avaliou a incidência de anafilaxia fatal por alimentos em menores de 19 anos de idade e mostrou ser 1,81 por milhão de indivíduosano, e segundo os autores, dez vezes menor que a por acidentes fatais ${ }^{32}$. Estudo norteamericano realizou análise retrospectiva de mortes, hospitalizações e visitas à urgência por anafilaxia induzida por alimentos, entre 2000 e 2014. Foram identificados 24 pacientes que faleceram em decorrência da anafilaxia, sendo que nenhum deles era menor de 4 anos de idade ${ }^{30}$. Estudos mais recentes e focados em anafilaxia no lactente não documentaram a ocorrência de óbito por anafilaxia entre os lactentes ${ }^{16,17,25}$.

Define-se como reação anafilática bifásica aquela em que há retorno dos sintomas após a resolução do quadro inicial sem que ocorra re-exposição ao fator desencadeante. Pouco se sabe sobre as causas que determinam a sua ocorrência. A incidência de reação bifásica tem sido identificada entre $1 \%$ e $20 \%$ das reações anafiláticas, e aproximadamente a metade delas ocorre nas primeiras 6 a 12 horas $^{9,34}$. Estudos pediátricos apontam que a reação bifásica ocorre em até $2 \%$ dos pacientes com anafilaxia induzida por alimento ${ }^{15}$.

\section{Quadro clínico}

A anamnese extensa é ponto fundamental no estabelecimento do diagnóstico de um paciente com suspeita de anafilaxia. É importante questionar-se sobre a ingestão de alimentos não usuais na dieta, uso de medicamentos e picadas de insetos ${ }^{1,7,9}$.

Apesar de os critérios clínicos diagnósticos da anafilaxia terem sido estabelecidos (Tabela 1) e se destinarem a todos os pacientes, independentemente de idade, nos lactentes merecem cuidado especial na sua avaliação e o diagnóstico de anafilaxia ainda é desafiador. A falta de capacidade do lactente em verbalizar parte das sensações experimentadas durante o episódio de anafilaxia (Tabela 3) ajuda na falha da caracterização mais clara do quadro e na sua subnotificação.
A análise de casuísticas de lactentes aponta que as manifestações mucocutâneas (variação entre 79\% e $100 \%$ ) seguidas das gastrointestinais (variação entre $26 \%$ e $89 \%$ ), respiratórias (variação entre $7 \%$ e $83 \%$ ), neurológicas (variação entre 12\% e $26 \%$ ) e menos frequentemente cardiovasculares (variação entre $0,9 \%$ e $21 \%$ ) são as descritas na anafilaxia do lactente (Tabela 2). Urticária (generalizada, rubor, angioedema), vômitos (persistente), tosse, sibilância, estridor, alterações de comportamento, assim como hipotensão e taquicardia são altamente sugestivos de anafilaxia no lactente ${ }^{12}$. Todavia, é importante reforçar a necessidade de acometimento de pelo menos dois sistemas do organismo para caracterizar o episódio de anafilaxia ${ }^{12}$. A Tabela 3 reúne os principais obstáculos (sinais e sintomas) para o diagnóstico clínico de anafilaxia no lactente.

A triptase é um mediador liberado por mastócitos e a sua quantificação sérica é útil quando o diagnóstico de anafilaxia é incerto. A dosagem de triptase sérica deve ser limitada ao episódio agudo, sobretudo nos momentos iniciais. A sua quantificação deve ser realizada entre 2 e 4 horas após o início dos sintomas e essa concentração deve ser interpretada com cautela, pois o lactente apresenta, normalmente, níveis mais elevados e que normalizam entre 9 e 12 meses de vida ${ }^{35}$.

\section{Diagnóstico diferencial}

Várias situações clínicas podem induzir ao diagnóstico equivocado de anafilaxia no lactente (Tabela 4). Como mencionado anteriormente, é essencial o acometimento de pelo menos dois sistemas do organismo para caracterizar o episódio de anafilaxia $^{12}$.

Diante de um lactente com suspeita de anafilaxia é preciso ser cuidadoso, pois infecções virais de vias aéreas podem ser acompanhadas de exantema, e de tosse e até mesmo sibilância se houver acometimento das vias aéreas inferiores, e assim mimetizar o quadro clínico de possível anafilaxia ${ }^{12,13}$. Vômitos e dores abdominais, nesta idade, também são multifatoriais e podem representar refluxo, obstipação ou outras etiologias não alérgicas ${ }^{12,13}$. A taquicardia pode ser secundária ao choro, a doença febril e ao desconforto respiratório ${ }^{12,13}$. Estes sintomas se associados à introdução de novos alimentos no início da vida, sobretudo os com alto risco de sensibilização, podem levar a diagnósticos equivocados de anafilaxia ${ }^{12,13}$. 
Tabela 3

Sinais e sintomas potenciais de anafilaxia em lactentes ${ }^{* 36}$

Sintomas que o lactente não descreve
Sinais potencialmente difíceis de interpretação e por quê?
Sinais potencialmente óbvios, mas não específicos

\section{Geral}

Sensação de calor, fraqueza, ansiedade, apreensão, desgraça iminente
Mudanças não específicas de comportamento, tais como choro persistente, agitação (pode também ocorrer com febre, hipertermia ou crises de choro)

\section{Pele e mucosas}

Prurido dos lábios, língua, úvula, ouvidos, garganta, nariz, olhos, e assim por diante; formigamento da boca e gosto metálico
Rubor (pode ocorrer também com febre, hipertermia ou crises de choro)
Urticária de início agudo (potencialmente difícil de diferenciar em lactente com dermatite atópica aguda; arranhaduras e escoriações, como tais, estarão ausentes em crianças pequenas); angioedema (face, língua, orofaringe)

\section{Sistema respiratório}

Congestão nasal, garganta apertada, aperto no peito, falta de ar
Rouquidão, disfonia (comum após crise de choro); salivação, aumento de secreções (comum em lactentes)
Tosse de início rápido, asfixia, estridor, sibilância, dispneia, apneia, cianose

\section{Trato gastrointestinal}

Disfagia, náuseas, dor/cólica abdominal
Cuspidor/regurgitação

(comum após alimentação), perda fecal (normal em lactentes especialmente se em amamentação); dor abdominal em cólica
Vômito abundante e repentino

\section{Aparelho cardiovascular}

Sensação de desmaio, pré-síncope, tontura, confusão, visão turva, dificuldade de audição, palpitações
Hipotensão (aferida com manguito aproriado), PA sistólica baixa para lactentes é definida com abaixo de $70 \mathrm{mmHg}$ para de 1 mês a 1 ano, e menos menos que $(70 \mathrm{mmHg}+$ [2x idade em anos]) nos primeiro e segundo anos de vida; taquicardia (acima de 120-130 batimentos/minuto do terceiro mês ao segundo ano de vida inclusive); perda de controle vesical ou intestinal (presente no lactente)
Pulso fraco, arritmia, sudorese excessiva, palidez, colapso/ inconsciência

\section{Sistema nervoso central}

Dor de cabeça

Sonolência

(comum no lactente após alimentação)

Perda rápida de sensibilidade, letargia ou hipotonia; convulsões

* Mais do que um órgão acometido. 


\section{Agentes etiológicos}

Os alimentos são os principais agentes etiológicos da anafilaxia no lactente ${ }^{15-18,20,24-30}$. Baseado nos dados apresentados na Tabela 2 verificamos que os alimentos envolvidos foram muito semelhantes nas diferentes casuísticas, havendo variações com relação à sua frequência de identificação. Destacam-se: leite de vaca (variação entre $16 \%$ e $67 \%$ ), ovo de galinha (variação entre $9 \%$ e 38\%), amendoim (variação entre $3 \%$ e $31 \%$ ), nozes (variação entre $3 \%$ e $9 \%$ ), trigo (variação entre $5 \%$ e $8 \%$ ), peixe (variação entre $5 \%$ e $6 \%$ ) e frutas (variação entre 3,2\% e 6\%) 15-18,20,24-30.

Em menor frequência são apontados medicamentos (antibióticos e anti-inflamatórios não esteroidais) e venenos de himenópteros. Devemos destacar também a alergia ao látex, sobretudo em pacientes com espinha bífida ou problemas urológicos e que foram submetidos a várias cirurgias ${ }^{1,7,9}$.

\section{Tratamento do episódio agudo}

Os vários documentos sobre anafilaxia publicados por sociedades médico-científicas, cujo objetivo é padronizar a abordagem dos pacientes com anafilaxia, não individualizam as recomendações de tratamento para a fase aguda da anafilaxia no lactente $e^{2,9,37,38}$. Apesar disso, Simons \& Sampson ${ }^{13}$ publicaram recomendações sobre a abordagem da anafilaxia direcionada ao lactente. Eles propõem 10 passos

\section{Tabela 4}

Diagnósticos diferenciais de anafilaxia no lactente ${ }^{13}$

Pele

Urticária, urticária pigmentosa/mastocitose, angioedema hereditário

Sistema respiratório (trato superior ou inferior) Obstrução congênita (prega laríngea, anel vascular, malácia) ou adquirida (aspiração de corpo estranho*, crupe, bronquiolite ou asma), asfixia, sufocação, respiração difícil

Trato gastrointestinal Obstrução congênita incluindo estenose hipertrófica de piloro e mal rotação ou adquirida incluindo enterocolite induzida por proteína alimentar (FPIES) com apresentação aguda e intussuscepção

Choque

Séptico, cardiogênico, hipovolêmico, distributivo

Sistema nervoso central

Convulsões, estado pós-ictal, trauma, abuso infantil, aumento da pressão intracraniana

Distúrbios metabólicos

Doenças infecciosas

Síndrome pertussis, gastroenterites, meningites

Ingestão de venenos/toxinas

Ingestão de alimento, droga, ou planta; overdose de drogas

Síndrome de Munchausen por procuração

Síndrome da morte súbita do lactente, evento com aparente risco de vida

Outro

* Os corpos estranhos aspirados são variáveis. Amendoim e nozes representam um terço dos corpos estranhos aspirados e também são agentes desencadeantes de anafilaxia. 
para o tratamento inicial da anafilaxia em lactentes, como se segue:

$1^{\circ}$ ter protocolo escrito de emergência para reconhecimento e tratamento da anafilaxia;

$2^{\circ}$ sempre que possível remover a exposição ao agente desencadeante, como por exemplo, interromper a infusão de medicação intravenosa;

$3^{\circ}$ avaliar as condições circulatórias, das vias aéreas, respiratórias, pele, além de obter o peso corporal do lactente;

$4^{\circ}$ solicitar imediatamente o auxílio de equipe de reanimação ou de médicos de serviços de emergência, em ambiente hospitalar ou não;

$5^{\circ}$ colocar o lactente em decúbito dorsal ou semireclinado em posição confortável nos braços do cuidador;

$6^{\circ}$ injetar epinefrina (adrenalina) solução milesimal (1:1000 ou $1 \mathrm{mg} / \mathrm{mL}$ ) intramuscular na face intermediária da coxa, na dose de 0,01 mg/ kg (máximo 0,3 mg) ou quando disponível por autoinjetor de adrenalina pediátrico $(0,15 \mathrm{mg})$;

$7^{\circ}$ se houver indicação, em qualquer tempo, iniciar a suplementação com $\mathrm{O}_{2}$ em alto fluxo (8 a $10 \mathrm{~L}$ ) através de máscara facial bem ajustada à face do lactente;

$8^{\circ}$ estabelecer acesso venoso e iniciar infusão de fluido com solução salina $0,9 \%$ inicialmente na dose de 10 a 20 mL/kg durante 5 a 10 minutos;

$9^{\circ}$ instituir, sempre que possível, o monitoramento eletrônico de parâmetros vitais: frequência cardíaca, respiratória e da pressão sanguínea. Monitorar a oxigenação empregando-se a oximetria de pulso;

$10^{\circ}$ quando indicado, realizar ressuscitação cardiopulmonar à frequência de 100 compressões torácicas por minuto, com profundidade de $4 \mathrm{~cm}$, e interrupções mínimas para realizar movimentos respiratórios na frequência de 15 a 20/minuto.

Os autores recomendam que esse protocolo para o tratamento inicial da anafilaxia seja afixado em local de grande visibilidade e ensaiado regularmente. Tão logo a anafilaxia seja diagnosticada, os $4^{\circ}, 5^{\circ}$ e $6^{\circ}$ passos devem ser realizados prontamente e de modo simultâneo ${ }^{13}$.

Vale comentar que a epinefrina é a medicação de eleição para o tratamento inicial da anafilaxia, apesar da ausência de estudos farmacológicos em lactentes, a sua administração é reforçada por todas as guias de tratamento, mas segue sendo subutilizada1,2,7,9,13,39. No Brasil, os autoinjetores de adrenalina não estão disponíveis, apenas em importadoras de medicamentos. Em geral são apresentados em duas dosagens: crianças $(0,15 \mathrm{mg})$ e adultos $(0,3 \mathrm{mg})$, por conta disso o autoinjetor na apresentação pediátrica é recomendado para crianças com peso superior a $10 \mathrm{~kg}$, embora haja relato de uso em lactentes com peso menor ${ }^{13}$.

Durante o tratamento inicial da anafilaxia nos lactentes, é infrequente a necessidade de mais de uma dose de epinefrina, o que reforça a sensação de que nessa população a anafilaxia é de menor gravidade. Topal e cols., ao avaliarem o tratamento de 104 crianças com anafilaxia em unidade de urgência, verificaram que nenhum lactente recebeu mais de uma dose de epinefrina, e que entre todas as crianças, 4,9\% recebeu duas doses de epinefrina, foram internadas em unidade de cuidados intensivos, e todas eram maiores de 12 meses $^{18}$. Outros dois estudos documentaram a necessidade de uma segunda dose de epinefrina por apenas um lactente avaliado em cada estudo ${ }^{16,25}$.

Embora anti-histamínicos e glicocorticosteroides sejam comumente administrados no tratamento de pacientes com anafilaxia, não devem ser usados como tratamento inicial ou como monoterapia, pois não revertem de modo rápido o edema de laringe, o broncoespasmo ou a hipotensão ${ }^{1,13}$. Para os pacientes que manifestam broncoespamo, a inalação de agente beta 2-agonista é importante, assim como a oferta de $\mathrm{O}_{2}$ caso a saturação periférica de $\mathrm{O}_{2}$ esteja abaixo de $95 \% 1,7,9$.

Os lactentes que não responderem de modo favorável ao tratamento inicial devem ser transferidos imediatamente a serviços pediátricos de emergência para terem atendimento especializado e melhor gerenciamento do quadro agudo ${ }^{13}$. Para os casos com boa evolução, observação mínima de seis horas no serviço de urgência é recomendada ${ }^{1,7,9}$.

A anafilaxia bifásica pode ocorrer em lactentes. As recomendações de tratamento farmacológico nessa faixa etária são extrapoladas de recomendações de tratamento para pacientes mais velhos ${ }^{13}$.

\section{Seguimento de longo prazo}

Após a alta do episódio agudo de anafilaxia recomenda-se que o paciente seja acompanhado, 
preferencialmente, por especialista em alergia/imunologia, para identificar e confirmar o agente etiológico e prevenir futuras recorrências ${ }^{1,2,9,13}$.

A educação dos responsáveis/cuidadores quanto à identificação precoce dos sinais e sintomas da anafilaxia, assim como sua evolução e o tratamento precoce são pontos fundamentais no acompanhamento destes pacientes. Os responsáveis/cuidadores devem ser munidos de um plano emergencial por escrito em que constem sintomas e sintomas iniciais capazes de orientá-los quanto ao reconhecimento precoce da anafilaxia e a instituição de tratamento adequado ${ }^{1,9,13}$.

Havendo disponibilidade dos autoinjetores de epinefrina (AIE), os responsáveis/cuidadores devem ser orientados e treinados como usá-los. No nosso meio os autoinjetores de epinefrina são medicamentos relativamente caros e possuem validade usual de 12 meses, havendo a necessidade de reposição frequente. Entretanto, a maioria dos estudos que avaliam a capacidade de retenção desses conhecimentos e a determinação para usá-los na urgência mostram resultados frustrantes, sobretudo se os responsáveis/ cuidadores não são da área da saúde. Estudo mostrou que apenas $29,9 \%$ de lactentes com anafilaxia grave foram tratados com injeções de epinefrina por seus cuidadores e têm como justificativas: não reconhecimento das reações, na disponibilidade de AIE, medo de administrar o medicamento, aguardar o aparecimento de mais sintomas e/ou não terem certeza sobre a necessidade de uso ${ }^{18}$.

\section{Identificação do agente desencadeante}

A investigação do agente etiológico da anafilaxia deve ter por base a história clínica. Considerando-se que no lactente predominam os alimentos como principais agentes desencadeantes, a pesquisa de $\lg \mathrm{E}$ específica in vivo e/ou in vitro é recomendada2,7,9,13.

A avaliação in vivo é realizada pelos testes cutâneos de hipersensibilidade imediata ( $\mathrm{TCHI})$ e pelos intradérmicos. A escolha dos alérgenos/alimentos a serem avaliados deve ser orientada pela história clínica do último episódio, e os TCHI devem ser realizados 4 a 6 semanas após o mesmo ${ }^{2,7,9,13}$. Com relação aos fármacos, há reagentes validados para os TCHI apenas para a penicilina, e para os outros antibióticos pode-se usar concentrações não irritativas da droga em estudo ${ }^{9,13}$. Na suspeita de participação de venenos de Hymenoptera, os TCHI podem ser realizados com alérgenos padronizados de abelha e vespa, no caso de formiga o extrato é de corpo total ${ }^{13}$. Os testes intradérmicos com alimentos não devem ser realizados e os com fármacos e/ou insetos são raramente executados ${ }^{13}$. Diante de história convincente, a presença de $\mathrm{TCH}$ negativo os pacientes devem ser re-testados semanas ou meses após ${ }^{13}$.

A determinação dos níveis séricos de IgE específica caracterizam a investigação in vitro. A depender dos alérgenos suspeitos, a análise dos componentes do alimento é importante no sentido de se estabelecer possível relação com gravidade da reação e a persistência de reatividade a esse alimento: por exemplo: caseína entre os alérgicos ao leite vaca, ovomucoide entre os alérgicos ao ovo de galinha, entre outros ${ }^{1,13}$. Com relação aos fármacos, poucos são os ensaios capazes de determinar a reatividade aos antibióticos, assim como aos venenos de himenópteros ${ }^{1,13}$.

Em pacientes submetidos a várias cirurgias ou procedimentos médicos, a anafilaxia pelo látex é possível. A presença de IgE específica a alérgenos da Hevea brasiliensis associada à história clínica confirmam o diagnóstico. Parte desses pacientes pode apresentar reação a determinados alimentos, tais como abacate, kiwi, banana, manga, melão, abacaxi, castanha ou avelã $\tilde{1}^{1,7,9}$.

É importante mencionar que a presença de $\mathrm{TCHI}$ positivo e a de IgE sérica específica a determinado agente indicam ser o paciente a ele sensibilizado, mas não são diagnósticos de anafilaxia 1,2,7,9,13.

Os testes de provocação com oferta de doses crescentes de agentes sob suspeita (alimentos), quando realizados, o devem ser em ambiente hospitalar, sob supervisão médica, executado por médico e profissionais de saúde treinados e experientes na abordagem de reações alérgicas graves. São importantes para descartar reatividade clínica de alérgenos relevantes ${ }^{13,15,40}$. Aos lactentes com anafilaxia induzida por fármacos, a avaliação deve se limitar à história clínica e testes cutâneos, embora os testes de provocação oral possam ser realizados ${ }^{13}$.

\section{Prevenção de recorrências}

A exposição aos alérgenos desencadeantes das reações e aos quais os lactentes estão sensibilizados e são clinicamente reativos deve ser evitada de modo irrestrito, pois é ponto fundamental na prevenção da recorrência da anafilaxia 1,7,9,13. Muitas vezes isso é desafiador para os cuidadores, sobretudo quando por 
tempo prolongado. Estudo longitudinal de pacientes (3 a 15 meses) com alergia a leite de vaca ou a ovo e acompanhados por tempo médio de 36 meses documentou que 53\% dos pacientes apresentaram reações por escapes e exposição ao alimento alergênico, sejam por não leitura de rótulos, contaminação cruzada ou exposição intencional do alimento realizada por terceiros ${ }^{26}$.

A literatura é farta em afirmar que a dessensibilização natural pode ocorrer em crianças com reatividade clínica a alguns alimentos, tais como leite de vaca e ovo de galinha. A presença de níveis baixos de $\lg E$ sérica específica e menor reatividade cutânea a esses alérgenos são indicativos de que esses pacientes perpassarão pela alergia alimentar. A tolerância a quantidades pequenas do alimento, ingeridas acidentalmente, na forma extensamente assada indica que o mesmo está caminhando para a tolerância ${ }^{13,41}$.

Para os pacientes com reação a fármacos e na impossibilidade de utilizar-se uma droga alternativa, a dessensibilização pode ser realizada por profissional médico experiente ${ }^{13}$. No caso de lactentes com anafilaxia induzida por picada de himenóptero, a recorrência das reações pode ser evitada com a implantação de imunoterapia com veneno padronizado de himenóptero ${ }^{13,17}$.

A anafilaxia seguida à vacinação para prevenção de doenças infecciosas é incomum, e os lactentes que a apresentarem deverão ser avaliados por alergista/ imunologista. A realização de $\mathrm{TCH}$ com a vacina sob suspeita e com os excipientes nela contidos tais como ovoalbumina, gelatina e neomicina é imperativa. Diante de um teste negativo, a vacina poderá ser administrada na dose e maneira usual. Caso o mesmo seja positivo, a vacina deverá ser administrada em doses fracionadas e crescentes, até atingir-se a dose total e o lactente mantido sob observação ${ }^{12,13}$.

Os lactentes com alergia ao ovo, mas sem história de anafilaxia ao ovo, poderão receber a vacina da Influenza por via intramuscular, pois a mesma tem conteúdo muito baixo de ovoalbumina na sua composição e a criança deverá ser mantida em observação por 30 minutos. Com relação à vacina da febre amarela, algumas vacinas têm quantidade de ovoalbumina superior à tolerada por pacientes alérgicos ao ovo. Nestes pacientes, a presença de IgE sérica específica a ovomucoide é marcador para que a vacina seja administrada em doses fracionadas e crescentes até atingir-se a dose total, e o paciente mantido sob observação por pelo menos 30 minutos $^{42}$.

\section{Considerações finais}

A anafilaxia no lactente é uma realidade apesar de especular-se ocorrer em menor frequência e gravidade nesses pacientes. Embora os critérios clínicos diagnósticos da anafilaxia estejam bem estabelecidos e se destinem a todos os pacientes, independentemente de idade, nos lactentes merecem cuidado especial. A falta de capacidade do lactente em verbalizar parte das sensações experimentadas durante o episódio de anafilaxia ajuda na falha da caracterização mais clara do quadro e na sua subnotificação. Os alimentos são os agentes etiológicos mais responsabilizados pelos episódios de anafilaxia nessa faixa etária, embora medicamentos e veneno de himenópteros também sejam mais raramente implicados. A adrenalina (epinefrina) intramuscular é o tratamento de primeira linha na fase inicial da anafilaxia, mas continua sendo subutilizada. Medidas de suporte à vida como reposição de fluidos, manutenção da permeabilidade das vias aéreas e suplementação com oxigênio também devem ser instaladas. Após a alta, o paciente deverá ser encaminhado para avaliação com alergista/imunologista para educação quanto à abordagem de novos episódios, e como evitar a sua recorrência.

\section{Referências}

1. Simons FE, Ardusso LR, Bilò MB, El-GamalYM, Ledford DK, Ring J, et al. World allergy organization guidelines for the assessment and management of anaphylaxis. World Allergy Organ J.2011;4(2):13-37. doi: 10.1097/WOX.0b013e318211496c.

2. Muraro A, Roberts G, Worm M, Bilò MB, Brockow K, Fernández Rivas M, et al. Anaphylaxis: guidelines from the European Academy of Allergy and Clinical Immunology. Allergy. 2014;69(8):1026-45. doi: 10.1111/all.12437.

3. Lieberman P, Nicklas RA, Oppenheimer J, Kemp SF, Lang DM, Bernstein DI, et al. The diagnosis and management of anaphylaxis practice parameter: 2010 update. J Allergy Clin Immunol. 2010;126:477-480.e1-42. doi: 10.1016/j.jaci.2010.06.022.

4. Sampson HA, Muñoz-Furlong A, Campbell RL, Adkinson NF Jr, Bock SA, Branum A, et al. Second symposium on the definition and management of anaphylaxis: summary report - second national Institute of allergy and infectious disease/food allergy and anaphylaxis network symposium. Ann Emerg Med. 2006;47:373-80. doi: 10.1016/j.annemergmed.2006.01.018.

5. ICD-11 for mortality and morbidity statistics. 4A84 Anaphylaxis. [Internet].Disponível em:https://icd.who.int/browse11/l-m/en\#/http:// id.who.int/icd/entity/1868068711. Acessado em: 24/05/2021.

6. ASCIA Anaphylaxis Clinical Update [Internet]. Disponível em:https:// www.allergy.org.au/images/stories/hp/info/ASCIA_HP_Clinical_ Update_Anaphylaxis_Dec2016.pdf.Acessado em: 21/05/2021.

7. Simons FER, Ardusso LFR, Bilò MB, Cardona V, Ebisawa M, ElGamal YM, et al. International consensus on (ICON) anaphylaxis. World Allergy Organ J. 2014;7(1):9. doi: 10.1186/1939-4551-7-9.

8. Turner PJ, Worm M, Ansotegui IJ, El-Gamal Y, Rivas MF, Fineman $S$, et al. Time to revisit the definition and clinical criteria for anaphylaxis? World Allergy Organ J.2019;12:100066. doi:10.1016/j. waojou.2019.100066. 
9. Cardona V, Ansotegui IJ, Ebisawa M, El-GamalY, Rivas MF, Fineman S, et al. World Allergy Organization anaphylaxis guidance 2020. World Allergy Organ J. 2020;13(10):100472. doi.org/10.1016/j. waojou.2020.100472.

10. Tanno LK, Chalmers R, Jacob R, Kostanjsek N, Bierrenbach $\mathrm{AL}$, Martin B, et al. Global implementation of the world health organization's International Classification of Diseases (ICD)11: The allergic and hypersensitivity conditions model. Allergy. 2020;75(9):2206-2218. doi: 10.1111/all.14468.

11. Wang Y, Allen KJ, Suaini NHA, McWilliam V, Peters RL, Koplin JJ. The global incidence and prevalence of anaphylaxis in children in the general population: a systematic review. Allergy. 2019;74:1063-80. doi:10.1111/all.13732.

12. Greenhawt M, Gupta RS, Meadows JA, Pistiner M, Spergel JM, Camargo CA Jr, et al. Guiding principles for the recognition, diagnosis, and management of infants with anaphylaxis: an expert panel consensus. J Allergy Clin Immunol Pract. 2019;7:1148-56. e5. doi: 10.1016/j.jaip.2018.10.052

13. Simons FE, Sampson HA. Anaphylaxis: Unique aspects of clinical diagnosis and management in infants (birth to age 2 years). J Allergy Clin Immunol. 2015;135(5):1125-31. doi: 10.1016/j. jaci.2014.09.014.

14. Robinson LB, Arroyo AC, Faridi MK, Rudders SA, Camargo CA Jr. Trends in US hospitalizations for anaphylaxis among infants and toddlers: 2006 to 2015. Ann Allergy Asthma Immunol. 2021;126(2):168-174.e3. doi:10.1016/j.anai.2020.09.003.

15. Tsuang A, Chan ES, Wang J. Food-induced anaphylaxis in infants: can new evidence assist with the implementation of food allergy prevention and treatment? J Allergy Clin Immunol Pract. 2021;9:57-69. doi: 10.1016/j.jaip.2020.09.018.

16. Ko J, Zhu S, Alabaster A, Wang J, Sax DR. Prehospital Treatment and Emergency Department Outcomes in Young Children with Food Allergy. J Allergy Clin Immunol Pract. 2020;8(7):2302-2309.e2. doi: 10.1016/j.jaip.2020.03.047.

17. Pouessel G, Jean-Bart C, Deschildre A, Van der Brempt X, Tanno LK, Beaumont $P$, et al. Food-induced anaphylaxis in infancy compared to preschool age: a retrospective analysis. Clin Exp Allergy. 2020;50:74-81. doi: 10.1111/cea.13519.

18. Topal E, Bakirtas A, Yilmaz O, Ertoy Karagol IH, Arga M, Demirsoy MS, et al. Anaphylaxis in infancy compared with older children. Allergy Asthma Proc. 2013;34:233-8. doi: 10.2500/aap.2013.34.3658.

19. Panesar SS, Javad S, de Silva D, Nwaru BI, Hickstein L, Muraro A, et al. The epidemiology of anaphylaxis in Europe: a systematic review. Allergy. 2013; 68(11):1353-61. doi: 10.1111/all.12272.

20. Solé D, Ivancevich JC, Borges MS, Coelho MA, Rosário NA, Ardusso $\mathrm{L}$, et al. Anaphylaxis in Latin American children and adolescents: the Online Latin American Survey on Anaphylaxis (OLASA). Allergol Immunopathol (Madr). 2012;40(6):331-5. doi: 10.1016/j. aller.2011.09.008.

21. Tanno L, Ganem F, Demoly P, Toscano C, Bierrenbach A. Undernotification of anaphylaxis deaths in Brazil due to difficult coding under the ICD-10. Allergy. 2012;67(6):783-9. doi: 10.1111/ j.1398-9995.2012.02829.x.

22. Nunes FA, Zanini F, Lima AM, Fernandes FR, Solé D, Wandalsen GF. Incidence, Triggering Factors, Symptoms, and Treatment of Anaphylaxis in a Pediatric Hospital in São Paulo, Brazil. Arq Asma Alerg Imunol. 2019;3(supl):S11.

23. Gawryjołek J, Krogulska A. Food-induced anaphylaxis in children up to 3-years-old - preliminary study. Allergol Immunpathol. 2021;49(3):56-64. doi:10.15586/aei.v49i3.77.

24. Jeon YH, Lee S, Ahn K, Lee SY, Kim KW, Kim HH, et al. Infantile anaphylaxis in Korea: a multicenter retrospective case study. J Korean Med Sci. 2019;34:e106. doi: 10.3346/jkms.2019.34.e106.

25. Samady W, Trainor J, Smith B, Gupta R. Food-induced anaphylaxis in infants and children. Ann Allergy Asthma Immunol. 2018;121:360-5. doi: 10.1016/j.anai.2018.05.025.

26. Fleischer DM, Perry TT, Atkins D, Wood RA, Burks AW, Jones $\mathrm{SM}$, et al. Allergic reactions to foods in preschool-aged children in a prospective observational food allergy study. Pediatrics. 2012;130:e25-32. doi: 10.1542/peds.2011-1762.
27. Rudders SA, Banerji A, Clark S, Camargo CA Jr. Age-related differences in the clinical presentation of food-induced anaphylaxis. J Pediatr. 2011;158:326-8. doi:10.1016/j.jpeds.2010.10.017.

28. Alvarez-Perea A, Ameiro B, Morales C, Zambrano G, Rodríguez A, Guzmán $M$, et al. Anaphylaxis in the Pediatric Emergency Department: analysis of 133 cases after an allergy workup. J Allergy Clinical Immunol Pract. 2017;5(5):1256-63. doi: 10.1016/j. jaip.2017.02.011.

29. Kahveci M, Akarsu A, Koken G, Sahiner UM, Soyer O, Sekerel BE. Food-induced anaphylaxis in infants, as compared to toddlers and preschool children in Turkey. Pediatr Allergy Immunol. 2020;31(8):954-61. doi: https://doi.org/10.1111/pai.13320.

30. Pistiner M, Mendez-Reyes JE, Eftekhari S, Carver M, Lieberman J, Wang J, et al. Caregiver-Reported Presentation of Severe FoodInduced Allergic Reactions in Infants and Toddlers. J Allergy Clin Immunol Pract. 2021;9:311-20.

31. Pumphrey RS, Gowland $\mathrm{MH}$. Further fatal allergic reactions to food in the United Kingdom, 1999-2006. J Allergy Clin Immunol. 2007;119:1018-9. doi: 10.1016/j.jaci.2007.01.021.

32. Umasunthar T, Leonardi-Bee J, Hodes M, Turner PJ, Gore C, Habibi $P$, et al. Incidence of fatal food anaphylaxis in people with food allergy: a systematic review and meta-analysis. Clin Exp Allergy. 2013;43:1333-41. doi: 10.1111/cea.12211.

33. Poirot E, He F, Gould LH, Hadler JL. Deaths, hospitalizations, and emergency department visits from food-related anaphylaxis, New York City, 2000-2014: implications for fatality prevention. J Public Health Manag Pract. 2020;26:548-56. doi: 10.1097/ PHH.0000000000001137.

34. Kraft M, Hofmeier KS, Ruëff F, Pföhler C, Renaudin JM, Bilò MB, et al. Risk Factors and Characteristics of Biphasic Anaphylaxis. J Allergy Clin Immunol Pract.2020;8(10):3388-3395.e6. doi:10.1016/j. jaip.2020.07.036. Epub 2020 Aug 4.

35. Belhocine W, Ibrahim Z, Grandne V, Buffat C, Robert P, Gras D, et al. Total serum tryptase levels are higher in young infants. Pediatr Allergy Immunol 2011;22:600-7. doi: 10.1111/j.1399-3038.2011.01166.x

36. Simons FE. Anaphylaxis in infants: can recognition and management be improved? J Allergy Clin Immunol. 2007;120(3):537-40. doi: 10.1016/j.jaci.2007.06.025.

37. Simons FE, Ebisawa M, Sanchez-Borges M, Thong BY, Worm M, Tanno LK, et al. 2015 update of the evidence base: World Allergy Organization anaphylaxis guidelines. World Allergy Organ J. 2015;8:32. doi: 10.1186/s40413-015-0080-1.

38. Shaker MS, Wallace DV, Golden DBK, Oppenheimer J, Bernstein JA, Campbell RL, etal. Anaphylaxis - a 2020 practice parameter update, systematic review, and Grading of Recommendations, Assessment, Development and Evaluation (GRADE) analysis. J Allergy Clin Immunol. 2020;145:1082-123. doi: 10.1016/j.jaci.2020.01.017.

39. Cardenas-Morales M, Hernandez-Trujillo V. Infant Anaphylaxis: A Diagnostic Challenge. Cur Allergy Asthma Rep. 2021;21:12. doi:10.1007/s11882-021-00990-4.

40. Bird JA, Groetch M, Allen KJ, Bock SA, Leonard S, Nowak-Wegrzyn $\mathrm{AH}$, et al. Conducting an oral food challenge to peanut in an infant. J Allergy Clin Immunol Pract. 2017;5:301-311.e1.

41. Solé D, Silva LR, Cocco RR, Ferreira CT, Sarni RO, Oliveira LC, et al. Consenso Brasileiro sobre Alergia Alimentar: 2018 - Parte 2 - Diagnóstico, tratamento e prevenção. Documento conjunto elaborado pela Sociedade Brasileira de Pediatria e Associação Brasileira de Alergia e Imunologia. Arq Asma Alerg Imunol. 2018;2(1):39-82. doi: 10.5935/2526-5393.20180005.

42. Cancado B, Aranda C, Mallozi M, Weckx L, Solé D. Yellow fever vaccine and egg allergy. Lancet Infect Dis. 2019;19(8):812. doi: 10.1016/S1473-3099(19)30355-X.

Não foram declarados conflitos de interesse associados à publicação deste artigo.

Correspondência:

Dirceu Solé

E-mail: dirceu.sole@unifesp.br 